\title{
XXXIX. On the $\alpha$ particles of radium, and their loss of range in passing through various atoms and molecules
}

\section{W. H. Bragg M.A. \& R. Kleeman B.Sc.}

To cite this article: W. H. Bragg M.A. \& R. Kleeman B.Sc. (1905) XXXIX. On the $\alpha$ particles of radium, and their loss of range in passing through various atoms and molecules, The London, Edinburgh, and Dublin Philosophical Magazine and Journal of Science, 10:57, 318-340, DOI: 10.1080/14786440509463378

To link to this article: https://doi.org/10.1080/14786440509463378

曲 Published online: 16 Apr 2009.

Submit your article to this journal $匚 \pi$

山ll Article views: 213

Q View related articles $\longleftarrow$

Citing articles: 473 View citing articles $\longleftarrow 3$ 
XXXIX. On the a Particles of Radium, and their Loss of Ranae in passing through various Atoms and Molecules. By W. H. BragG, M.A., Elder Professor of Mathematics and Physics in the University of Adelaide, and R. Klemman, B.Sc., Demonstrator *

TN previous papers which we have contributed to this Magazine $\dagger$ we have shown that the a particle moves always in a rectilinear course, spending its energy as it traverses atoms of matter, until its velocity becomes so small that it cannot ionize and there is in consequence no further evidence of its motion. Each $x$ particle possesses therefore a definite range in a given medium, the length of which depends on the initial velocity of the particle and the nature of the medium. Moreover, the $\alpha$ particles of radium which is in radioactive equilibrium can be divided into four groups, each group being produced by one of the first four radioactive changes in which a particles are emitted. All the particles of any one group have the same range and the same initial velocity.

The present paper may be regarded as a continuation of the papers cited. Its contents are arranged under the following heads :-

1. Improvements in the apparatus used for measuring the ranges and relative strength of the four groups of rays.

2. Results of experiments with the new apparatus giving the following values of the ranges in air at a pressure of $76 \mathrm{~cm}$. and a temperature of $20^{\circ} \mathrm{C}$.

$\begin{array}{ll}\text { Radium ............ } & 3.50 \mathrm{~cm} . \\ \left.\begin{array}{l}\text { Emanation or } \\ \text { Radium A. }\end{array}\right\} \ldots . . . & 4.23 \\ \begin{array}{l}\text { Radium A or } \\ \text { Emanation. }\end{array} \text {...... } & 4 \cdot 83 \\ \text { Radium C .......... } & \mathbf{7} \cdot 06\end{array}$

These are correct, we believe, to $.05 \mathrm{~cm}$. The pressure and temperature are stated, since a change of $1 \mathrm{~cm}$. in the pressure or $5^{\circ} \mathrm{C}$. in the temperature would produce an effect which could be observed. We have not yet determined which of the two middle groups belongs to the Emanation and which to $\mathrm{RaA}$; but we have constructed a special piece of apparatus which will, we hope, settle the point.

* Communicated by the Authors. Read before the Royal Society of South Australia, June 6, 1904.

† Phil. Mag. Dec. 1904. 
The results also show that the four groups are alike in all respects save that of initial speed; and further, that the a particle spends its energy at a rate which is approximately inversely proportional to the square root of its speed.

3 . Determinations of the loss of range of $\alpha$ particles in consequence of their passage through various substances, from which it appears that for all the materials examined the loss in traversing any atom is nearly proportional to the square root of the weight of that atom. The loss in the case of a complex molecule is proportional to the sum of the square roots of the weights of the constituent atoms.

4. Discussion of these results.

\section{$\$ I$.}

In the apparatus which we first used for these experiments we found that our measurements were liable to considerable irregularities. The most troublesome of these was an occasional sudden and violent increase of the ionization current which would cause the electrometer spot to fly off the scale. This would occur in the middle of a period of satisfactory working. It appeared to be due to the liberation of emanation from the radium under observation, and we have therefore enclosed the whole of our apparatus in a metal vessel through which a current of dry air is made to pass continually.

In other respects the apparatus now used differs but little from that described on p. 728 of the Phil. Mag., Dec. 1904: except that we have added an arrangement by which an aluminium screen can be drawn over the radjum so as to cut off all $\alpha$ radiation, and we have made it possible to set the ionization-chamber at any desired depth. Both these arrangements can be worked from the outside of the enclosing vessel. It is necessary that the air which is drawn through should be fairly dry. In this country a difference of $30^{\circ} \mathrm{F}$. between the wet and dry bulb thermometers is not unusual, and at these times artificial drying is hardly necessary; but we find it more satisfactory to draw the air at all times through a set of large calcium-chloride tubes, the current passing day and night during the progress of any series of experiments. The need for dryness appears to arise from the fact that moist air acts on the thin film of radium which we employ and liberates emanation.

At one time we endeavoured to secure the complete removal of any liberated emanation by passing the whole stream of air directly over the radium just before it left the vesse?; 
but with this arrangement the remaining moisture of the air seemed to do more harm in liberating emanation than the current did good by carrying it away. We now pass the current of dry air through the vessel from one end to the other; but the radium film probably foels little draught, being protected by the set of vertical metal tubes which stand over it (see the previous papers). When this is done the leak which the electrometer shows, the radium being out of range, is very small compared with the principal leak and is fairly constant. In the experiments described later it amounted usually to one or two millimetres in ten seconds, whilst the principal leak might be as much as sixteen centimetres in the same time.

This residual leak is due to various causes. One of them is the leak which takes place from the metal of the ionizationchamber, and the wire which connects it with the electrometer, to the earthed metal case which screens the chamber and its connexions from external influence. This current is supplied by the ions which are continually formed by various agents in the air within the earthed casing, and is driven by the Volta potential-differences between the metals of the connexions and the casing. It can be materially altered by changing the material of the casing, or part of it, e.g. exchanging zine for copper. If a speck of radium is placed within the casing so as to increase the supply of ions the effect is surprising. As an illustration we allowed the electrometer connexions to end in a brass wire $15 \mathrm{~cm}$. long, which projected horizontally in the open air, the ionization apparatus being disconnected. When a large earthed copper tube containing the radium speck was placed so that the brass wire was along its axis, the electrometer showed a large deflexion, whereas, when the copper was displaced by zinc, the deflexion was also large, but in the opposite direction.

The whole of the apparatus including the keys which connect the electrometer to earth or the ionization-chamber is enclosed in earthed metal casing. The keys are worked by electromagnets which are fixed externally to the casing, and operate on iron armatures within. We have found it necessary to be careful as to the contacts of these keys. Want of perfect contact canses incomplete charging or discharging of the electrometer quadrants, shown by kicks and irregular movements of the light-spot when the keys are used. We have obtained good results by making the contacts of platinum surfaces, so arranged as to rub each other at the make or break. This does not introduce any want of definition into the beginning or ending of a leak, since both 
these times are determined by the breakings of contacts, and these are done by the electromagnets with great suddenness.

An arrangement which we find to be of great importance, is the ion-trap which is placed under the gauze of the ionization-chamber. 'The space below the gauze B, forming the lower plate of the ionization-chamber $\mathrm{AB}$, is of course
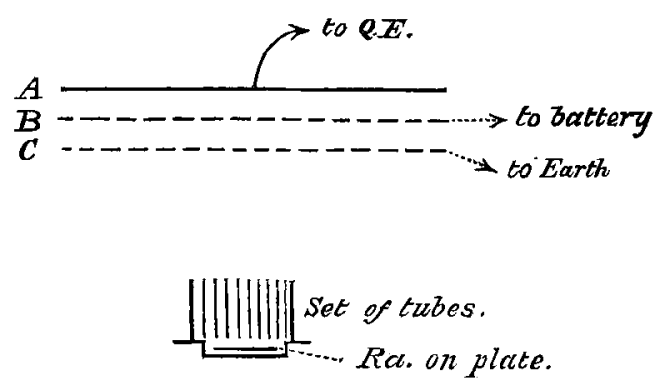

filled with the ions formed by the rays which cross it. When the gauze $\mathrm{C}$ is not present, then although $\mathrm{B}$ is at a high potential, some of these ions manage to cross it and add themselves to those in the chamber $\mathrm{AB}$, thus disturbing the results to be measured. We were first aware of this effect occurring in any marked degree when we began to use a platinum dish on which had been evaporated a few drops of water containing a speck of very pure radium bromide. $\mathrm{We}_{\mathrm{e}}$ were so very fortunate as to be presented by Mr. Soddy with a milligramme or two of this material; by its use our experiments were very greatly improved, as will be described a little later. On the first occasion of its use, however, we were puzzled by a rather curious result. The radium and the tubes above it were rather close up to the gauze B. We were measuring the various leaks as, millimetre by millimetre, we pushed the radium still closer. Every now and then we drew the aluminium screen over the radium so as to discover how much of what was observed was really due to the $\alpha$ rays. We found that after the screen had been so used and withdrawn, the proper leak was materially lessened, but recovered its old value in a short time. When the screen was drawn over again, the leak (which was then not due to a rays) fell instantly to its usual very small value, and again, when the screen was withdrawn, the proper leak was for a time too small. We guessed therefore that some of the leak, with the screen off, was due to ions made outside the chamber, which afterwards drifted into it. The interposition of the aluminium 
screen instantly cleared all the space between itself and the gauze B of ions, because the gauze was at a high potential and the screen was earthed. No more ions were made in this space until the screen was withduawn. When this was done, the space just below the gauze and above where the screen had been was momentarily empty of ions, and though the continued passage of the a rays through it soon refilled it, yet there was a short time during which the ions in the space were below normal value, and therefore the drift upwards into the ionization-chamber was small. If this drift existed, it was certainly to be avoided, and we therefore placed below the gauze $B$ a parallel gauze $C$ about three millimetres away, which was put to earth. No ions could cross the powerful field which existed between the two gauzes. The second gauze cut off a number of $\alpha$ rays which would otherwise have entered the chamber, but there were still quite enough. With this new arrangement the irregularity described above has disappeared completely. Another irregularity has also disappeared at the same time; it was probably due to the same cause, and removed in the same way. It occurred when one or other of the bundles of rays was just out of range of the ionization-chamber, and took the form of a small leak which seemed to prelude the arrival of the real rays; it formed a slight projection on the ionization curve just above the true and large increase which represented the entrance of the rays of the bundle into the ionizationchamber, as the radium was pushed higher and nearer. It often blurred the corners of the ionization curves, and made it more difficult to determine the exact ranges of the rays.

The electrometer used in these experiments is of the Dolezalek pattern. It has been been frequently calibrated, either by charging it and causing it to share its charge with an auxiliary condenser, or by measuring a given radium leak, first, when the electrometer was in parallel with the condenser, and again when it was not. The deflexion for a given charge is wonderfully constant over a wide range of voltages of the needle; though the deflexion for a given potential varies considerably. It is not found satisfactory to maintain the conductivity of the quartz fibre by dipping it in a solution of calcium chloride; in dry weather it does not conduct. It is better to recharge the needle to 250 volts every few hours, if it is used for so long; the needle may then leak considerably without alteration of the deflexion for a given charge. 
$\S$ II.

Having made these various improvements in our apparatus, and become more familiar with its use, we made a new layer, as already mentioned, from the pure radium bromide which Mr. Soddy had so kindly sent us. This at once gave good results. The curves shown in fig. 1 show the considerable precision which it was now possible to attain.

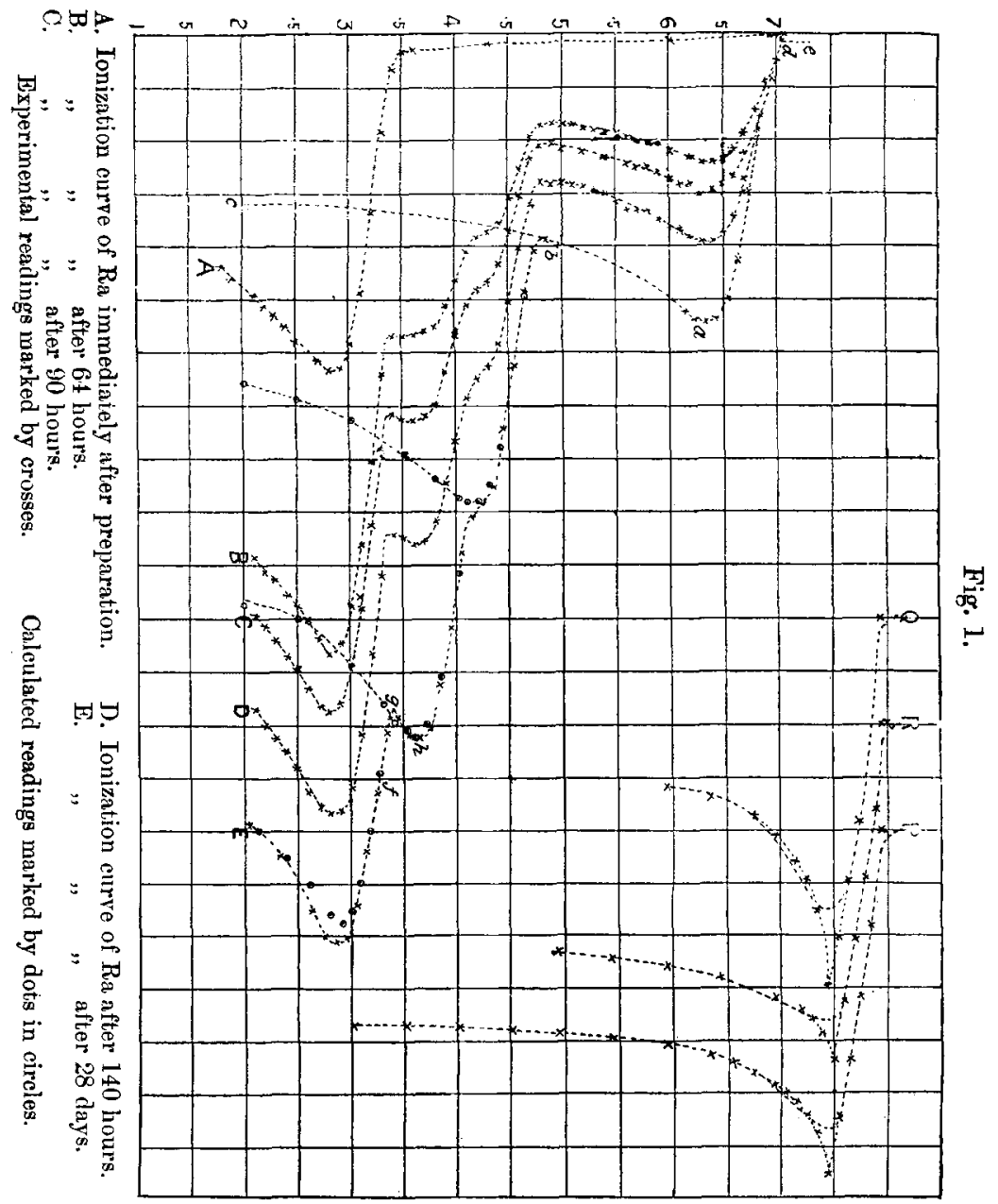

It may be well to repeat here that these ionization curves show the amount of the ionization due to the $\alpha$ rays at various distances from the radium layer, the medium ionized being 
air at ordinary pressures and temperatures. A bundle of copper tubes is placed in a vertical position over the horizontal layer, so that only such $\alpha$ particles as move in a direction nearly vertical are allowed to proceed upwards. The bundle used at present contains about 100 tubes made of thin copper, each $2 \mathrm{~mm}$. in diameter and $1 \mathrm{~cm}$. long. Tho ionization-chamber consists of an upper horizontal plate of :luminium, and a lower plate of brass gauze; the distance between the plates in most of the experiments described in this paper was set at two millimetres. The ordinates represent the distances from the radium plate to the gauze; and the abscissæ the leaks on the quadrant electrometer. The absolute value of these leaks was not accurately measured, as there was no object in knowing more than their relative values; but it was calculated that the greatest leak measured was about $10^{-11}$ ampere.

Curve A, in fig. 1 , shows the ionization due to the a rays when the dish was first prepared. It had been kept at a bright red heat for some minutes after preparation. The shape of the curve shows that all radioactive products except the radium itself had been completely removed. On comparison with the similar curve on p. 730 of the paper already cited, it will be seen that the new radium layer is thinner than that from which our first results were obtained; for the maximum value of the ionization occurs at $2.85 \mathrm{cms}$. instead of at $2 \cdot 5$. The greatest range is about $3 \cdot 4$ as before. This means that the a particles from the bottom of the new layer lose, in coming up through the material of the layer, a range of $5.5 \mathrm{~mm}$. measured in air : the corresponding amount for the older layers was $9 \mathrm{~mm}$. The new layer is nevertheles: nearly twenty times stronger than the old. The term layer is, in fact, rather inappropriate. Actually the radium is not distributed over the dish in a thin uniform sheet but is studded over the surface in the form of small crystals which can be easily seen under the microscope. Consequently a weaker solution of radium bromide does not give, when evaporated, a thinner layer; but simply a smaller number of crystals. The various bundles, and in particular those from Ra, Emanation, and $\mathrm{RaA}$ do not therefore stand out any better from each other when a weaker solution is evaporated, as we found on making the experiment. But the greater purity of the new layer gives the result which we formerly sought to obtain by evaporating very weak solutions. Presumably the crystals are now more regularly arranged, and are not overlaid by other matter which acts as a screen.

Since the radiation of the new layer is so strong, the 
residual errors of the experiment become relatively small. In particular, consecutive readings of the same leak become much more nearly in agreement than before; when the readings are large, the difference between two such readings is generally less than one per cent. of the whole. For small readings it is proportionately greater; and it is quite clear that much of this irregularity is due to some extraneous cause, and is not due to variability of the principal leak, or inaccuracy either of the observer or his apparatus. Other experimenters bave made a similar observation.

In the case of all the measurements recorded in this paper, the current was allowed to run into the electrometer for ten seconds; the mean of the first and second points of rest of the needle was taken as the correct reading. This was justifiable, as relative, not absolute, measurements were wanted; and as the difference between these two measurements was very nearly proportional to the whole deflexion.

Curves B, C, and D in fig. 1 show the state of the radium at definite intervals after preparation of the layer. Similar curves were published in our previous paper, but the curves now shown are much more accurate, and supply much more information. In the first place, the division of the $\alpha$ rays into four groups is now seen very clearly, and the range of each group can be measured accurately. In the former curves, the separation of the two central groups was not well effected, but now it is plain; the improvement being mainly due to the greater thinness and evemness of the radium layer.

In the same figure, curve $\mathrm{E}$ shows the state of the radium when 28 days old. According to Rutherford's theory the number of a particles in each group is the same, provided the radium preparation is old enough to be in radioactive equilibrium. This can be proved from the curve $\mathrm{E}$ in the following manner.

The curved side $a b$, belonging to $\mathrm{Ra} \mathrm{C}$, is produced to $c$, as shown. The line $d e$ represents a certain ionization produced by $\beta$ and $\gamma$ rays which is intercepted by the aluminium screen. It is the small difference between the readings at (say) $7.5 \mathrm{~cm}$. when the aluminium is on and off. When the radium is within range, and the ionization of the a rays is measured as the difference between the on and off readings, this small quantity of $\beta$ and $\gamma$ ionization is included in the difference. Its value must be nearly constant over all ranges, as it is a certain fraction of the total $\beta$ and $\gamma$ ionization, which latter is found to be constant by experiment. We may therefore 
consider the figure bounded by the vertical line through $d e$ and by the curved line $d a b c$ to represent the ionization by the Ra $\mathrm{C}$ particles over the whole of their course except the first two centimetres.

This enrve is now added to itself, being first lowered through $2.23 \mathrm{~cm}$, and the points represented by dots in circles represent some of the results of the addition. It will be seen how nearly these points lie on the actual ionization curve, on which the experimental readings are marked by crosses. Again the curve $d a b c$ is lowered, by $6.0 \mathrm{~mm}$. this time, and added on to the sum already obtained, and the new points which show the result of the addition are also marked as dots in circles. These also lie very nearly on the ionization curve. For the last time the curve is added on, being this time lowered $7 \cdot 3 \mathrm{~mm}$.; and again the calculated points lie on the experimental carve except just at the peak. Thus the full curve is formed by the superposition of four simple curves, each alike in all respects but that of height; and the inference is that the four groups of $\alpha$ particles are alike in every respect but that of initial velocity. The differences of the ranges are in this way given with more accuracy than that with which the actual ranges can be found, for it is hard to avoid all uncertainty about the latter because the gauze of the ionization-chamber is not easily made quite flat. If the curve $f g$ is produced to meet the curve $h g$ also produced, then the meeting-point is at a height $3.4 \mathrm{~cm}$. This point should represent the arrival of the Ra rays at the middle of the ionization-chamber, which is $1 \mathrm{~mm}$. higher than the gauze. Hence the range of the particles from $\mathrm{R}_{\mathrm{a}}$ is $3.50 \mathrm{~cm}$.; and the ranges of the other three groups are $4 \cdot 23,4 \cdot 83$, and 7.06 . These must be correct to $0.5 \mathrm{~mm}$.

Since the ionization curve can now be drawn with some accuracy, it becomes reasonable to make an attempt to discover the relation between the velocity of the $a$ particle and the rate at which it spends its energy.

Let $v$ be the velocity of the particle, and $s$ the distance it has yet to run. Suppose that the energy spent per $\mathrm{cm} .=k \cdot v^{-n}$, where $k$ and $n$ are constants. We seek a value for $n$.

$$
\text { Then } \begin{aligned}
\frac{1}{2} m \frac{d v^{2}}{d s} & =k v^{-n} . \\
\therefore \quad m v^{n+1} d v & =k d s \\
\frac{m v^{n+2}}{n+2} & =k s .
\end{aligned}
$$


Hence the rate of expenditure of energy on ionization

$$
\begin{aligned}
& =\frac{k}{v^{n}} \\
& \times \frac{1}{s^{n+2}} .
\end{aligned}
$$

If the strip of air (supposed thin) in which the ionization is measured is at such a height above the radium layer that the $\alpha$ rays from the top of the layer pass it by a distance $x$, and those from the bottom by a distance $x-d$, then the ionization produced in this strip by the $\alpha$ particles from all parts of the layer

$$
\begin{aligned}
& \propto \int_{x-d}^{x} x^{-\frac{n}{n+2}} d x \\
& =\mathrm{A}\left(x^{\frac{2}{n+2}}-(x-d)^{\frac{2}{n+2}}\right),
\end{aligned}
$$

where $A$ is a constant. If the rays from the top of the layer pass the strip by $x$, and those from the bottom do not reach it, then the ionization

$$
=A x^{\frac{2}{n+2}} .
$$

In the curves as found experimentally the effect is further complicated by the fact that the ionization-chamber has an appreciable depth of $2 \mathrm{~mm}$., and that the cones of rays have an appreciable vertical angle. We need not, however, take these factors into account in the formula. We can give $n$ different values, plot the corresponding curves as found from the formula, and round off the corners in arcordance with the effect which the two disturbing factors must have.

When this is done, it is found that $n=\frac{1}{2}$ gives a curve which is very near to the actual form. Choosing the value $5 \mathrm{~mm}$. for $d$, which seems proper from an inspection of the curve and from trial, the ionization is equal to $\mathrm{A} x^{\frac{4}{5}}$ from $x=0$ to $x=5 \mathrm{~mm}$, and thereafter to $\mathrm{A}\left\{x^{\frac{1}{5}} \cdot-(x-5)^{\frac{4}{5}}\right\}$. The curve obtained from this formula is plotted in fig. $1, P$; and the corners are rounded off by the dotted line. The value of $A$ is chosen so as to make the curve more easily comparable with the experimental curve $\mathrm{E}$ (portion $d a b c$ ).

Curves $Q$ and $R$ are derived from the formula when $n$ is put equal to 1 and to $1 / 3$ respectively. It will be seen that they do not approach the experimental form so well, and err in opposite directions. 
The conclusion is that the a particle spends its energy at a rate which is approximately inversely proportional to the square root of its velocity.

\section{$\S$ III.}

From the results described above as well as from those described in the previous papers (Phil. Mag., Dec. 1904) it will be clear that the law of the absorption of the $\alpha$ rays is considerably different from that which has been generally assumed hitherto. It has been supposed that a stream of radiation gradually diminishes in power as it penetrates any material substance, the rate of loss being approximately expressible by an "absorption coefficient," and the law being therefore nearly exponential. It is true that it has been pointed out that this coefficient is not constant for any one stream in any one gas, but varies with the distance of the stream from the source. If this variation were small, there might be some yround for considering that the law was fundamentally dependent on an exponential form, but varied from it on account of some secondary cause. As a matter of fact, howover, the variations are far too great to permit of such a supposition.

The true state of affairs is very different. There is no such absorption coefficient, nor any approach to an exponential law.

Each $\alpha$ particle pursues a rectilinear course, no matter what it encounters: it passes through all the atoms it meets, whether they form part of a solid or a gas (or in all probability of a liquid), suffering no deflexion on account of any encounter until, at any rate, it is very near the end of its course. It loses energy as it goes, a portion of this at least being spent in producing ions. This drain on its energy, at least, robs it of its extraordinary powers of penetration and ionization.

From this it will be clear that there are two quite independent characteristics of a stream of a radiation : one, the number of a particles in it, the other, the energy of their motion. The latter may more or less be evenly distributed amongst the various particles. It is possible for the latter of these characteristics to vary considerably whilst the first does not change at all. For example, in the curves described above, the $\alpha$ particles from $\mathrm{Ra} C$ remain unchanged in number up to nearly $6.5 \mathrm{cms}$. from the commencement of their motion; but their energy has almost vanished at the longer distance. Again, as will be seen later, a thin metal plate 
may be placed in the way of the stream, and so rob every particle of some of its energy, but not a single one is brought to rest by collision with the atoms of metal, and the number of particles in the stream remains unchanged.

In our experiments two different quantities are measured, one the range of the $a$ particles, the other, the ionization which they produce. The range of the $\alpha$ particle in a given substance is the distance it can penetrate before losing its power of making ions in that substance, which can be collected and measured by electrical means. We assume that when it ceases to have this power, the energy has fallen to an amount which is very small compared to its initial energy (say 1/500), so that even if it varies in different substances, such a variation cannot affect the range appreciably. As a matter of fact, it is probable that no such variation exists.

The range of an $\alpha$ particle in any substance is therefore dependent on the original energy of projection. It is not quite proportional to it, because, as we have pointed out already, the a particle does not spend energy at exactly the same rate at all periods of its flight; towards the end of its range its rate of expenditure is somewhat increased. If the particle is made to pass through some material other than air, its range may be much altered. For example, if it passe; through a thin silver film whose thickness is $-001 \mathrm{~cm}$. its range in air, supposing it to be allowed to complete its course in that medium, is diminished by more than $3 \mathrm{cms}$. Or again, the $\alpha$ particle which has a range of $7.0 \mathrm{cms}$. in air has only a range of $3.3 \mathrm{cms}$. in methyl bromide at the same pressure and temperature. Consequently, if we measure the range of the $\alpha$ particles in different gases, or after their passage through thin films of matter, we obtain a measure of the comparative rates at which energy is expended by a particle in passing through these gases and materials.

The second quantity which is measured by us is tho ionization produced by the particle at various distances from its source. From this measurement we are able to discover, amongst other things, whether the stream has suffered in any other respect than that of loss of range when it has passed through thin material films, supposing that the rest of the flight is in ordinary air ; and if we compare the quantities as measured in different gases, we are enabled to compare tho conductivities which are imparted to these gases by $\alpha$ particles moving through them under conditions otherwise similar.

These two quantities are independent of each other. It is true that if the conductivity of a gas were proportional to the 
number of ions produced by the particle, and that if the total number of ions produced by an $\alpha$ particle were the same, no matter the gas in which it completed its course, then the product of the ranges and the ionization per $\mathrm{cm}$. of path would be a constant. Now Rutherford has made measurements of the total ionization produced by the particle in some substances, and found it the same in each case. But there are other substances for which this is not true; e.g., ether, ethyl chloride, and methyl bromide ; and in consequence the second of these suppositions is not justified. It is even possible that the first may also prove incorrect. It is conceivable that the a particle may spend energy on ionization whilst passing through a molecule, especially a complex molecule, and that the products may never get away from the molecule so as to be separated by the electrical field, and measured by the electrometer. That this actually occurs certainly seems a natural inference from some of the following results.

Let us now consider the experimental evidence. In fig. 2 are drawn three curves, $\mathrm{A}, \mathrm{B}$, and $\mathrm{C}$. The first of these represents the ionization curve of radium in air. Curve $\mathrm{B}$ shows the result of placing a number of thin sheets of silver-leaf over the radium, so that the rays had to traverse these before reaching the ionization-chamber. Curve $\mathrm{C}$ shows the effect of substituting thin silver-foil for the leaf. The product of the density and the thickness was in the case of the leaf .00213 , and in the case of the foil 00967 . These figures were readily obtained by weighing a measured area of the material in each case; the leaf was not easy to bandle, and the loss of range in its case not very great, so that the foil experiment is the more reliable.

The curves A B C are clearly similar in shape, and differ only in their height above the zero line. The silver sheet has not in either case caused any modification of the $\alpha$ stream, except that it has cut off an equal amount from the range of each particle.

If some of the particles had been stopped by the metal, or if some had lost more energy than others, there would have been more or less distortion of the curve. But the former condition does not occur, as bas been already explained, and the latter was also prevented in this case by the great uniformity of the foil, which was polished on both sides.

The loss of range in the case of the silver-foil is $33.5 \mathrm{~mm}$. If we take the density of the air to be $\cdot 0012$, we may say that a silver stratum for which thickness $X$ density $=\cdot 00967$ is equivalent to an air stratum for which the same product $=3 \cdot 35 \times \cdot 0012=\cdot 00402$. The ratio of these quantities is $2 \cdot 41$. 
In fig. 2 are shown the results of some of our experiments of this kind on other metal films. All do not show the same regularity as the silver: for we have found great difficulty in obtaining uniform films in the majority of cases. The

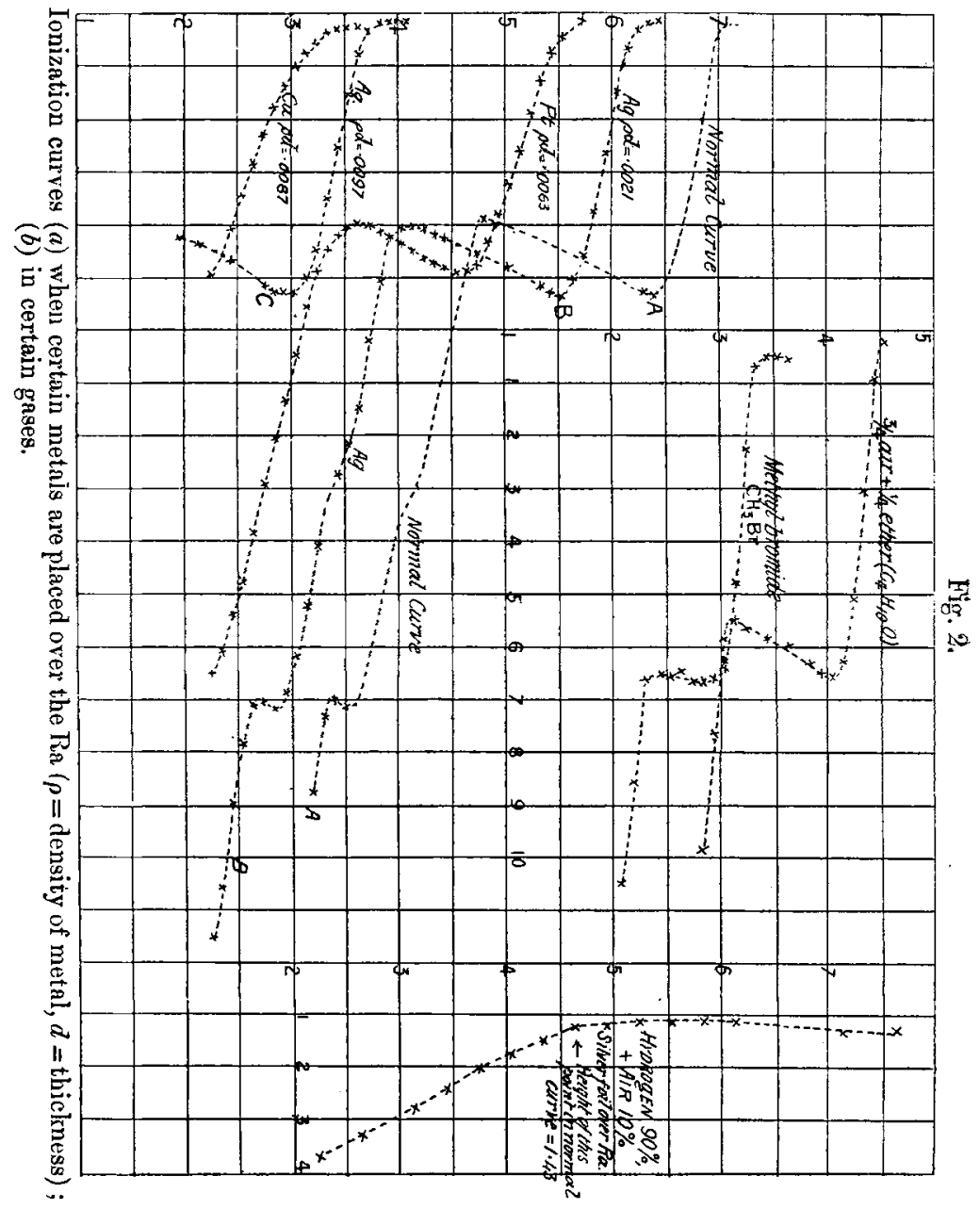

rolls used by the jewellers are not fine enough to give perfect results, and we believe there is no goldbeater in Australia at present. Nevertheless the metal workers have managed to roll for us films of gold, platinum, and copper which have given us fair results; aluminium leaf was procurable and 
thin tinfoil. When a film is not uniform, the fact is made evident by a distortion of the curve, e. g. in the case of copper. It will be seen that the top of it slopes too much, implying that the $a$ rays do not come into the ionizationchamber as suddenly as they ought to do. Some have, in fact, been less checked than others because they have passed through thinner portions of the somewhat uneven film. We have measured, in such cases, the drop of the curve as shown by its amount at the middle point of the top of the $\mathrm{RaC}$ portion. E. g., in the case of platinum we have measured the depth of the middle point of the top of the curve below that of the middle point of the top of the normal curve.

The results of these experiments are shown in the following table. The first column of figures gives the product of the thickness of the metal film and its density, the second the corresponding drop of the curve, multiplied by the density of air, and the third the ratio of these two products.

\begin{tabular}{|c|c|c|c|c|c|}
\hline & I. & II. & III. & IV. & Ratio IV./IIX. \\
\hline Gold & $\cdot 0121$ & .00396 & 3.05 & $14 \cdot 2$ & $4 \cdot 65$ \\
\hline Platinum ...... & .00633 & .00192 & 829 & $14 \cdot 0$ & $4 \cdot 25$ \\
\hline $\operatorname{Tin} * \ldots \ldots \ldots$ & .0051 & $\cdot 00212$ & $2 \cdot 41$ & $10 \cdot 85$ & 4.50 \\
\hline Silver & $\cdot 00967$ & $004 \cup 2$ & $2 \cdot 41$ & $10 \cdot 4$ & $4 \cdot 30$ \\
\hline Copper .......... & .00873 & .00492 & $1 \cdot 78$ & $7 \cdot 96$ & $4 \cdot 45$ \\
\hline Aluminium ... & .00258 & .00209 & $1 \cdot 23$ & $5 \cdot 15$ & $4 \cdot 20$ \\
\hline
\end{tabular}

* Tinfoil contains a certain proportion of lead; but this could not affect the result very appreciably.

It is remarkable that the numbers in the third column are nearly proportional to the square root of the atomic weights of the metals. To bring this out more clearly, the square roots are shown in a fourth column, and in a fifth the ratios of the numbers in the two previous columns.

Moreover, air itself falls approximately into line with the metals. Its ionization ratio should be entered in the third column as unity, and the average square root of its atomic weight as $(4 \sqrt{ } 14+\sqrt{ } 16) / 5=3 \cdot 79$. The corresponding entry in the last column should be also $3 \cdot 79$.

Also it is easily seen that hydrogen is not far away from the others. For it has been shown by Strutt that its conductivity under ionization as compared with air is 226 . Assuming for the present that this means that a $1 \mathrm{~cm}$. layer of hydrogen has the same effect as a $\cdot 226 \mathrm{~cm}$. layer of air, then the ratio of the product of the thickness and the density in the case of the hydrogen to the similar product in the case of the air is $1 / 14 \cdot 4 \times \cdot 226=\cdot 31$. The square root of its atomic weight is 1 , and $1 / \cdot 31=3 \cdot 2$. 
Since the atomic weight is of such consideration in this law, it is more direct to state the results in another way. Instead of comparing the stopping powers of strata of metal and air of equal weights, we can compare the stopping powers of strata containing equal numbers of atoms, and therefore the stopping powers of individual atoms. For example, a stratum of silver produces the same effect as a stratum of air whose weight is $2 \cdot 41$ times greater. Thus for equal weights silver stops $1 / 2 \cdot 41$ times as much as air; but for equal numbers of atoms it stops $108 / 14 \cdot 4 \times 2 \cdot 41$ times as much as air. This ratio, which is equal to $3 \cdot 11$, may be called the "stopping power" of the silver atom, referred to the air atom as a standard. The latter is an imaginary atom having an atomic weight $14 \cdot 4$, a molecular weight $28 \cdot 8$, and an atomic square root $3 \cdot 79$.

The stopping powers of the various metals examined will be set out below, in conjunction with those of certain gases.

When we found that a simple law seemed to cover the behaviour of substances differing so widely in all their properties as those enumerated, we thought it advisable to examine such other substances as were available. We were unable to obtain other metal films; and we therefore turned our attention to gases. Now no striking evidence for the square-root law could be obtained from an examination of gases whose atomic weights were nearly the sume as that of air, such as oxygen or nitrogen. Indeed, it had already been shown by Strutt that their behaviour was very much the same as that of air. Nevertheless, it was not to be forgotten that their proved properties were not against the square-root law.

We therefore made experiments with the following gases, which contained atoms whose weights were very different. from those of air atoms:-

Methyl bromide, methyl iodide, ethyl chloride, carbon tetrachloride, ether, and hydrogen.

Methyl bromide was a most suitable gas for our purpose. The ratio of its molecular weight to that of air is $3 \cdot 2 s$. If, then, the loss of range in passing through this gas were proportional to the density, the range of the particle from $\mathrm{RaC}$ would be only about $7 / 3 \cdot 28$ or $2 \cdot 3 \mathrm{~cm}$., provided the gas were at atmospheric pressure. But if the square-root law were true, the range would be much greater, and could be calculated thus :-

The carbon atom should contribute a stopping power proportional to $\sqrt{ } 12$, the three hydrogen atoms to $3 \sqrt{ } 1$, and the bromine atom to $\sqrt{ } 80$. Total $3 \cdot 46+3+8 \cdot 95=15 \cdot 41$. In air the stopping power should be the average of that of Phil. Mag. S. 6. Vol. 10. No. 57. Sept. 1905. 
four molecules of nitrogen and one of oxygen. The stopping power of nitrogen should be proportional to $2 \sqrt{ } 14$ or $7 \cdot 48$, that of oxygen to $2 \sqrt{ } 16$ or 8 . Now $(4 \times 7 \cdot 48+8) / 5=7 \cdot 58$. Therefore the stopping power of the $\mathrm{CH}_{3} \mathrm{Br}$ molecule as compared to that of air should be $15 \cdot 41 / 7 \cdot 58=2 \cdot 03$; and the range of the a particle of $\mathrm{RaC}$ should be $7 / 2 \cdot 03=3 \cdot 4 \mathrm{~cm}$.

A sufficient quantity of methyl bromide was prepared and kept liquid in a freezing-mixture. The vessel containing our apparatus was joined to an air-pump and exhausted till the remaining pressure was equal to about $6 \mathrm{~cm}$. of mercury. The liquid was then allowed to pass in under atmospheric pressure until the vessel was full of the gas. The pressure was again reduced to $i \mathrm{~cm}$., and vapour introduced until the pressure in the vessel was once more equal to that of the atmosphere.

The ionization curve was then obtained. It is plotted in fig. 2, and shows that the range is nearly as calculated from the square-root law.

It was not advisable to take it for granted that the vessel contained vapour only; and after a little time we hit upon a method of determining this point satisfactorily. The method also proved particularly useful in the case of mixtures of air and vapours, as will be described presently. Immediately after measuring the range of the particle in the gas, we opened a communication between the vessel and an exhausted globe of about one litre capacity. The capacity of the vessel itself was 6 litres. The weight of the gas so drawn over was compared with the weight of the air that came over under similar conditions when the larger vessel contained air only; and from this ratio the proportion of air and vapour could be deduced. For example, the weight of the mixture of air and methyl bromide was 2.599 grams. The corresponding weight of air was $\cdot 800$ gram. (It was not, of course, at atmospheric pressure.) Hence if $x=$ the ratio of the number of air molecules to the number of molecules of $\mathrm{CHBr}$,

$$
\begin{aligned}
\frac{x+3 \cdot 28}{x+1} & =\frac{2 \cdot 599}{\cdot 500}, \\
& =3 \cdot 25, \\
\therefore \quad x & =1 / 75 .
\end{aligned}
$$

The extreme range of the $\alpha$ particle of $\mathrm{RaC}$ in $\mathrm{CH}_{3} \mathrm{Br}$ is shown by the curve to be $3 \cdot 32$. This is the distance from the radium to the gauze of the ionization-chamber. The 
latter was $2 \mathrm{~mm}$. deep, and half this, viz. $1 \mathrm{~mm}$., should be added in order to get the true range, which may therefore be taken as $3 \cdot 42$. The corresponding range in air is $7 \cdot 06$.

Hence, if $x=$ stopping power of the methyl-bromide molecule,

$$
\begin{aligned}
\frac{1+75 x}{76} & =\frac{7 \cdot 06}{3 \cdot 42}=2 \cdot 07, \\
\therefore x & =2 \cdot 09,
\end{aligned}
$$

which is very nearly the value already calculated on the squareroot law. The errors of experiment were not so small that this almost absolute agreement can be looked on as more than accidental. It will be seen, however, that all the gases examined gave good results.

The curve of a gas is different in some important respects from that which is obtained in examining the loss of range in metal films. In the latter case, all the ordinates are reduced from those of the normal air-curve by nearly the same amount; in the former, they are reduced in nearly the same proportion. Again, in the case of the metal film (provided it is uniform) the abscissae are unaltered ; in the case of the gas, the abscissa are all altered in the same proportion and show the amount of ionization produced by the a particle in traversing the gas. For example, the extreme abscissa of the Ra C group has the value 666 in the gas and 547 in air ; thus the ionization per $\mathrm{cm}$. is $666 / 547$ or 1.27 times greater in the gas than in the air. Since, however, the range is 2.07 times shorter, the ratio of the total ionization in the gas to the total ionization in air is only $1 \cdot 27 / 2 \cdot 07=62$ nearly.

In most of the complex gases examined the a particle produces less ionization than in air. We propose to make a further examination of this point.

In the case of the other gases, the procedure was very similar. Carbon tetrachloride, methyl iodide, and ether are liquid at ordinary temperatures and pressures, and in their case we introduced the necessary quantity of liquid to give a vapour pressure of about two-thirds that of saturation, and then filled up with air. We fonnd it better to do this than to attempt the experiment with low pressures in our vessel, for the latter bad so many joints and stuffing-boxes that it would not keep a vacuum very well. It was, hotwever, quite airtight enough to retain a mixture unchanged for hours provided it was at atmospheric pressure. The proportion of air to vapour was afterwards determined in the manner already described. 
Ethyl chloride was tried before we used the method of weighing a definite quantity of the gas. But the vessel was emptied and filled three times, and there must have been very little air in it. It cannot give a decisive answer as to the validity of the square-root law, because, as it happens, the square-root law and the simple density law give nearly the same value. On that account we have not yet repeated the experiment.

Hydrogen presented some special difficulties. The range (on the square-root law) of the a particle of Ra C must be about $30 \mathrm{~cm}$., and our apparatus only measures up to $8 \mathrm{~cm}$. We therefore placed a piece of silver-foil over the radium, which was equivalent to $3.3 \mathrm{~cm}$. of air. This cut down the range of the $\mathrm{RaC}$ particles to 3.63 , and the range of the swiftest of the others to 1.43 . The former when multiplied by four would still be out of range, but the latter would be within it.

Fig. 2 (p. 331) shows that this was the case. With a mixture of hydrogen and air in the proportion (by pressure) of 9 to 1 it was at $4 \cdot 6$. With mixtures containing a greater proportion of hydrogen it rose somewhat, but a small proportion of air made little relative difference. The curve is of course very much drawn out vertically, and drawn in horizontally.

The results obtained for all the above substances are collected in the following table:-

TABLE, showing "stopping power" of various atoms and molecules as compared with that of air. The atomic weight of the supposititious atom of air is taken as $14 \cdot 4$, and the "atomic square root" is $3 \cdot 79$.

\begin{tabular}{|c|c|c|c|}
\hline & \begin{tabular}{|} 
Stopping power \\
of atom or \\
molecule.
\end{tabular} & $\begin{array}{l}\text { Ratio of } \\
\text { square } \\
\text { roots. }\end{array}$ & $\begin{array}{l}\text { Ratio of atomic } \\
\text { or molecular } \\
\text { weights. }\end{array}$ \\
\hline Hydrogen ......... & 246 & 265 & .069 \\
\hline & & & 1 \\
\hline Aluminium ... & 1.53 & $1 \cdot 3 \mathrm{~S}$ & 1.88 \\
\hline Copper ........ & $2 \cdot 42$ & $2 \cdot 1$ & 4533 \\
\hline Silver & $3 \cdot 11$ & 275 & $7 \cdot 5$ \\
\hline Tin. & 342 & 288 & $8 \cdot 2$ \\
\hline Platinum & $4 \cdot 12$ & $3 \cdot 7$ & $13 \cdot 5$ \\
\hline Gold $\ldots \ldots \ldots \ldots \ldots$ & 4.45 & $3 \cdot 7$ & 137 \\
\hline Metbyl bronlide ... & 209 & $2 \cdot 09$ & 328 \\
\hline Ethyl chloride ............. & 230 & $2 \cdot 3 i$ & 2.23 \\
\hline Methyl iodide ............ & $2 \cdot 49$ & $2 \cdot 35$ & $4 \cdot 9$ \\
\hline Ether $\ldots \ldots \ldots$ & $3 * 30$ & 368 & $2 \cdot 56$ \\
\hline Carbon tetrachloride & 38 & $3 \cdot 61$ & $5+41$ \\
\hline
\end{tabular}


These results are shown graphically in fig. 3 , in which the ordinates and abscissæ represent the numbers in the first

Fìg. 3.

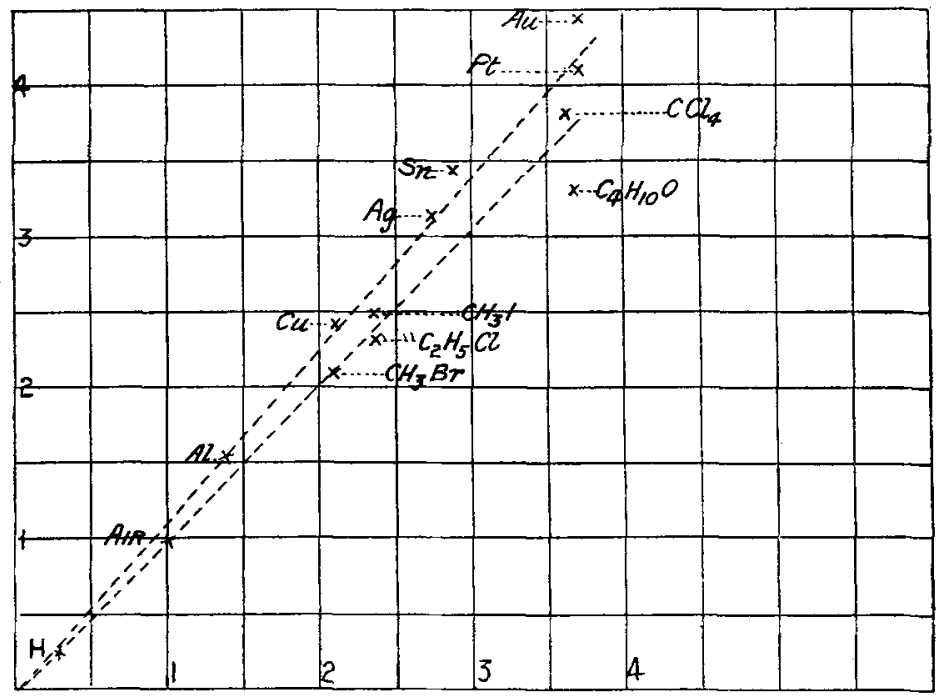

and second columns respectively. It is curious that the metals and gases seem to lie on rather different lines. Possibly this is due to the difference between solid and gas; possibly again to the fact that the gases tested have such complex molecules. Further research may make the point clearer. Several other points arose in the course of the experiments, which deserve further inquiry. For instance, the metal films did not cause exactly the same drop throughout all the curve; the slower a particles were a little less affected than the swifter.

\section{$\S I V$.}

From these risults it appears that the following law is true for certain elements, at least to a first approximation.

The energy spent by an a particle in its passage through an atom is proportional to the square root of the atomic weight. It can never be said that this is a law which is general to all the elements until all the elements have been experimented upon. In the case of many of them, the experimental difficulties will be very great. Since, however, the law holds with some accuracy in several cases, it becomes desirable to consider whether any physical interpretation can be placed upon it. 
Now the energy which is spent by the $\alpha$ particle is certainly spent, in part, in producing ions. If the rate of the expenditure of the energy as a whole is related to the atomic weight by so simple a rule as that of the square root, it is probable that the part which is spent on jonization obeys the same rule. For if not, and if the expenditure on ionization follows some other law, then the remaining expenditure must follow such a law that the two together compound into the simple rule of the square root. It is proper to put aside this more complicated hypothesis until we have considered the simpler one.

We may therefore advance the law one stage and state it thus :-The energy spent on ionization by an $\alpha$ particle in its passage through an atom is proportional to the square root of the atomic weight. The question now arises: Is the variation from atom to atom due to a difference in the number of ions produced, or in the energy required to produce an ion, or in both these quantities? Because an oxygen atom absorbs four times as much energy as a hydrogen atom, are there four times as many ions produced from an oxygen atom, but the energy per ion the same; or is the act of ionization four times as difficult, but the number of ions the same; or are neither of these suppositions true, and is the difference between oxygen and hydrogen in this respect due to a variation in each of the two quautities?

Now the total conductivity imparted to a gas by the passage of a particle through it is very nearly the same for the simpler gases. This is trne even though some of them differ widely from each other in their atomic weights, e.g. oxygen and hydrogen. From this it appears probatile that the energy required to produce an ion is always the same. It is true that in some of the complex gases the total conductivity is less than in the simpler gases, but this can be explained on the hypothesis that the same number of jons is made in these heavy molecules, but that they find a difficulty in escaping from the molecule and are recombined, so that they add nothing to the conductivity of the gas. If this hypothesis is not entertained, we are driven to suppose that the energy required to make an ion is not the same in some of the complex molecules, and that the number made varies by just so much as to make the total loss of energy of the particle in each molecule follow the same simple law as holds for the simpler molecules. For the present at least this complicated supposition must also be set aside, and again we may make an advance in the statement of our law. It now stands :-

The nunber of ions made by an $\alpha$ particle in passing 
through an atom is proportional to the square root of the atomic weight.

It should, perhaps, be pointed out that this does not mean that the $\alpha$ particle makes the same number of ions out of each atom of the gas through which it is passing; but merely compares the average per atom. It asserts, for example, that in going through oxygen the a particle makes a number of ions which is four times as many as the number made by the particle moving with the same speed in passing through hydrogen at the same pressure and temperature.

It is possible that this rule implies that a particle must make more than one pair of ions in passing through any one atom, unless ionization is only rarely the consequence of the encounter. For if only one pair could be made in each atom, and if the a particle made one pair in crossing each atom of hydrogen, then in oxygen at the same pressure and temperature as the hydrogen, only the same number of ions would be made per centimetre, which is not the case. Nor does it appear possible to suppose that ionization does not occur at a large proportion of encounters; the number of ions made is of the same order as the number of atoms traversed, according to Rutherford.

The most reasonable interpretation of these results seems therefore to be (1) that the a particle makes the same number of ions during its course no matter what the gas which it traverses; $(2)$ that the energy required to make a pair of ions is always the same; and (3) that the observed variations in the conductivities in some cases are due to the failure of ions to get free from the molecule in which they are made.

Under these circumstances we must look for the origin of the square-root law in some disposition which limits the number of ions that can be made in an atom. It is not to depend on any variation of the energy required to make a pair of ions; but must be due to some condition which only gives the $\alpha$ particle such an opportunity of making ions as is to be measured by the square-root of the atomic weight; and, where it forbids the act of ionization, forbids also the corresponding expenditure of energy.

We can only make guesses as to what this condition may be. It has been maintained, on good evidence (see Meyer, 'Kinetic Theory of Gases,' \$112), that the atom has a disk-like form, and that the various atoms and molecules differ in two dimensions only. It is possible that an explanation of the square-root law may be found in the hypothesis that ions can only be formed on the circumference of the atom's disk, for the amount of that portion of the atom 
which lies on the circumference is proportional to the square root of the whole. Under such circumstances an $\alpha$ particle, if it struck the atom anywhere except at the edge, would pass through without result.

Since the energy required to make a pair of ions is probably the same no matter what the atom or molecule may be in which they are made, and no matter whether it be solitary or a constituent of a complex molecule, we seem driven to the belief that ionization consists in breaking bonds which are always the same, both in character and in strength. The electron is torn from its union with something else which is always the same, and must form some constituent of all atoms. This hypothesis also makes reasonable the supposition that an act of ionization may be performed within a complex molecule, and yet no conductivity be imparted to the gas. For the act would then consist in the breaking of an electron from its attachment to this common constituent, and would not of necessity imply separation from the system of the molecule. The idea of a common constituent is, of course, an old one. It is certainly strengthened by the discovery that radium and its successive products emit $a$ particles which are exactly alike.

We wish to express our gratitude to Mr. J. P. V. Madsen, B.Sc., for the generous assistance which he gave us during several weeks of full work, and to Professor Rennie, D.Se., and Mr. A. J. Higgin for their kindness in making the chemical preparations.

XL. On the Space occupied by Atoms: The Theories of Th. W. Richards and J. Traube. By Prof. Dr. J. Traube, Berlin*.

GOR several years $\dagger$ I have been busy with investigations regarding the space relations of different forms of matter, and have repeatedly pointed out the extraordinary importance of this much neglected domain of science.

To my satisfaction, so distinguished an investigator as Th. W. Richards has recently $\ddagger$ taken up the same problem,

* Communicated by the Author.

+ See, especially, my latest work, Drude's Ann. d. Phys. Bd. v. p. 548 (1901), and Bd. viii. p. 267 (1902); further, the account in the Boltwmann-Festschrift, p. 430 (1904); also Zeitschr. f. anorg. Chem. Bd. xxxiv. p. 413 (1903), Bd. xxxriii. p. 399 (1904), Bd. xl. p. 372 (1904); and the older account: "Ueber den Raum der Atome, F. W. A hrens, Samml. chem, techn. Vorträge, Bd. iv. p. 1 (1899), and my Grundriss der Physikal. Chemie (Enke, Stuttgart, 1904).

t Th. W. Richards, Zeitschr. f. Physil. Chem. Bd. xi. pp. 169 \& 602 (1902); Bd. xlii. p. 129 (1903); and Bd. xlix. pp. 1 \& 15 (1904). 